\title{
From Microscopic to Macroscopic Description of Composite Thin Panels: A Road Map for Their Simulation in Time Domain
}

\author{
Luis Diaz Angulo $^{\circledR}$, Miguel Ruiz Cabello, Jesus Alvarez, Member, IEEE, \\ Amelia Rubio Bretones, Senior Member, IEEE, and Salvador G. García ${ }^{\circledR}$, Senior Member, IEEE
}

\begin{abstract}
In this paper, we show a simulation strategy for composite dispersive thin-panels, starting from their microscopic characteristics and ending into a time-domain macroscopic model. In a first part, we revisit different semianalytic methods that may be used to obtain the S-parameter matrices. The validity of them is assessed with numerical simulations and experimental data. We also include some formulas that may be used to tailor the shielding effectiveness of panels in a design phase. In a second part, we present an extension to dispersive media of a subgridding hybrid implicit-explicit algorithm finite difference time domain (FDTD) devised by the authors to deal with that kind of materials. The method, here presented and applied to the FDTD method, is a robustly stable alternative to classical impedance boundary condition techniques. For this, a previous analytical procedure allowing to extract an equivalent effective media from $\mathrm{S}$-parameters is presented, thus making this road map able to simulate any kind of dispersive thin layer. A numerical validation of the algorithm is finally shown by comparing with experimental data.
\end{abstract}

Index Terms-Finite difference time domain (FDTD), implicitexplicit schemes, subcell models, thin-layer modeling, time domain (TD).

\section{INTRODUCTION}

$\mathbf{T}$ HE ADEQUATE simulations of the electromagnetic (EM) properties of composite multilayered complex thinpanels are of extreme importance, since they are part of modern materials used, e.g., in the automotive or aeronautic industries. The challenge emerges because of the multiple scales which are involved, being impossible in practice to mix microscopic and macroscopic geometrical features in

Manuscript received July 28, 2017; revised October 27, 2017; accepted December 17, 2017. Date of publication January 4, 2018; date of current version February 5, 2018. This work was supported in part by Spanish MINECO, EU FEDER under Project TEC2013-48414-C3-01, Project TEC2016-79214C3-3-R, and Project TEC2015-68766-REDC, in part by J. de Andalucia, Spain under Grant P12-TIC-1442, in part by Alhambra-UGRFDTD (AIRBUS DS), and in part by the CSIRC alhambra.ugr.es supercomputing center. This paper is an expanded version from the IEEE MTT-S International Conference on Numerical Electromagnetic Modeling and Optimization for RF, Microwave, and Terahertz Applications, May 17-19, 2017, Seville, Spain. (Corresponding author: Luis Diaz Angulo.)

L. Diaz Angulo, M. Ruiz Cabello, A. R. Bretones, and S. G. García are with the Department of Electromagnetism, University of Granada, 18071 Granada, Spain (e-mail: Imdiazangulo@ugr.es; mcabello@ugr.es; arubio@ugr.es; salva@ugr.es).

J. Alvarez is with Airbus Defence and Space, 28906 Getafe, Spain (e-mail: jesus.g.alvarez@airbus.com).

Color versions of one or more of the figures in this paper are available online at http://ieeexplore.ieee.org.

Digital Object Identifier 10.1109/TMTT.2017.2786263 a single simulation. A typical workflow to approach this type of problems can be sectioned in two stages: the EM modeling of the thin-panel properties and the incorporation of this model into a numerical solver. In a first stage, our concern is to obtain an accurate EM characterization of the thin-panel transmission and reflection properties for normal incidence as a function of frequency. We can distinguish three distinct procedures to obtain these parameters: to perform experimental measurements with standard setups such as ASTM D4935 or its variants [1]-[3], to derive analytical formulations accounting for its microscopic structure [4]-[6], or to carryout simulations of the unit cell with appropriate periodic boundary conditions [6]-[8]. These results can be typically expressed in two frequency-dependent matrix forms: transmission matrices $(A B C D)$ and S-parameters, both linked by well-known equivalence relations [9].

In a second stage, the treatment of the obtained S-parameters depends on the method to be used by the solver. A common approach to this problem is to treat each thin-panel by impedance boundary conditions (IBCs) to avoid costly fine meshings of the material structure. In the frequency domain (FD), the introduction of IBCs is similar to classic BCs such as perfect electrically/magnetically conducting, and the macroscopic parameters can be used with little or no treatment, directly modifying the BCs on the element surfaces accordingly. In time domain (TD), on the contrary, these parameters cannot be used directly and require the extraction of appropriate dispersive models. Specifically within the framework of the finite difference time-domain (FDTD) method, a typical approach consists of using a technique known as network IBC (NIBC) [4], [10]. For this, a partial fraction expansion, in terms of complex-conjugate pole-residue pairs, is performed by fitting the frequency-dependent $S$-parameters, e.g., by using a vector-fitting (VF) technique [11]. However, the poles need to be carefully chosen to avoid instabilities, and, even in this case, late-time instabilities are reported to appear because of the noncollocated nature of FDTD [12], [13].

To overcome these limitations, in [14], we proposed a novel technique based on a robustly stable subgridding boundary condition (SGBC), which does not present the late-time instabilities of NIBC. The method was based on a hybrid implicitexplicit (HIE) combination of the classical FDTD with a Crank-Nicolson time-domain (CNTD) unconditionally stable method. However, the formulation presented in [14] required to know the multilayer interior structure of the thin panel, 
in terms of its bulk conductivity and the thickness of each layer. As a consequence, that approach could not be used to model arbitrarily dispersive thin-panels, and it has been extended to this end.

In this paper, we extend the contribution made in [15] and present a full road map which starts from the S-parameters, found either by simulation or by semianalytical methods and finishes with a dispersive set of constitutive parameters that can be efficiently simulated by FDTD by using the SGBC method. The rest of this paper is organized as follows. In Section II, we present a discussion on the available analytical models for the panels and discuss which ones are preferable for different configurations. We also present a discussion on the factors that dominate the SE behavior of the panels which may be useful, for instance, to decide on the optimal materials, densities, or shapes of a fiber. To validate these analytical models, in Section IV, we crosscompare with results from simulations of their microscopic structure. In Sections V and VI, we briefly describe how to find a suitable TD model in terms of constitutive parameters, and we extend the SGBC method to deal with arbitrarily dispersion by generalizing the unconditionally stable CNTD to dispersive media. Finally, we present a simple validation with experimental data where we have followed the whole road map described in this paper.

\section{Thin PANel Modeling}

Let us start our discussion by defining some basic concepts that will help us to fix a notation. When a harmonic planewave, having an incident electric field amplitude $E^{i}$, illuminates perpendicularly a flat panel, part of it is reflected $\left(E^{r}\right)$, and part of it gets transmitted through it $\left(E^{t}\right)$. In consequence, thin panels can be regarded as multiport networks, identifying port 1 as the side of the panel illuminated by the plane-wave and port 2 as the side where the wave is transmitted

$$
\left[\begin{array}{l}
E_{2} \\
H_{2}
\end{array}\right]=[\Phi]\left[\begin{array}{l}
E_{1} \\
H_{1}
\end{array}\right]=\prod_{i=1}^{N_{l}}\left[\Phi_{i}\right]\left[\begin{array}{l}
E_{1} \\
H_{1}
\end{array}\right]
$$

with $\Phi$ and $\Phi_{i}$ being the transmission $(A B C D)$ matrix of the thin panel and of the stacked layers composing it, respectively. The scattering parameters can be derived from matrix $\Phi$, using, for instance, the relations given in [9].

For panels in free-space, characterized by an intrinsic impedance $\eta_{0}$, we can obtain the transmission coefficient as

$$
T=S_{21}=\frac{2 \eta_{0}}{\Phi_{11} \eta_{0}+\Phi_{12}+\Phi_{21} \eta_{0}^{2}+\Phi_{22} \eta_{0}} .
$$

For anisotropic media, we have to account for $\mathrm{TE}_{z}$ and $\mathrm{TM}_{z}$ polarizations, henceforth simply TE and TM. We can identify four transmission matrices for TE and TM conversions

$$
\left[\begin{array}{l}
E_{2}^{\mathrm{TE}} \\
H_{2}^{\mathrm{TE}} \\
E_{2}^{\mathrm{TM}} \\
H_{2}^{\mathrm{TM}}
\end{array}\right]=\left[\begin{array}{ll}
{\left[\Phi_{\mathrm{TE} \rightarrow \mathrm{TE}}\right]} & {\left[\Phi_{\mathrm{TE} \rightarrow \mathrm{TM}}\right]} \\
{\left[\Phi_{\mathrm{TM} \rightarrow \mathrm{TE}}\right]} & {\left[\Phi_{\mathrm{TM} \rightarrow \mathrm{TM}}\right]}
\end{array}\right]\left[\begin{array}{l}
E_{1}^{\mathrm{TE}} \\
H_{1}^{\mathrm{TE}} \\
E_{1}^{\mathrm{TM}} \\
H_{1}^{\mathrm{TM}}
\end{array}\right]
$$

and regard them as four independent two-port networks, one for each of the possible mode conversions. Proceeding with

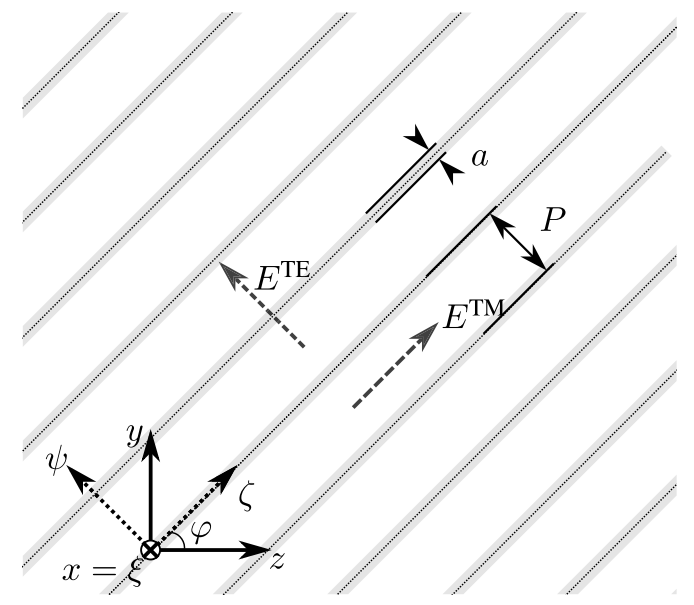

Fig. 1. Geometrical characteristics of the wire array: diameter $a$ and period $p$. Wire mesh 2 -D representation with local $(\xi, \psi, \zeta)$ and global $(x, y, z)$ coordinate systems.

each of them in a similar way as for isotropic media, we can obtain the four S-parameters for inter- and intramodal transmission coefficients.

A typical parameter often used is the shielding effectiveness (SE), as a measure of the capability of the panel to shield an enclosure from the EM environment. It is defined as the inverse of the transmission coefficient

$$
\mathrm{SE}=\frac{1}{T}=\frac{\left|E^{i}\right|}{\left|E^{t}\right|} .
$$

Though the method presented in the following can in general find the S-parameters, we will henceforth just focus in the SE. Note that (4) is a scalar and sufficient for isotropic media. However, for anisotropic media, a TE wave can have transmitted TM and TE components, $T_{\mathrm{TE} \rightarrow \mathrm{TM}}$ and $T_{\mathrm{TE} \rightarrow \mathrm{TE}}$, respectively, and we can define an average SE by

$$
\mathrm{SE}_{\mathrm{av}}=\frac{2}{\left|T_{\mathrm{TM} \rightarrow \mathrm{TM}}\right|+\left|T_{\mathrm{TE} \rightarrow \mathrm{TE}}\right|} .
$$

\section{Periodic Materials Based on Conductive Meshes}

Modern composite materials consist often in multilayered structures including woven/nonwoven carbon fibers (CFC), reinforcing wire-meshes, solid or expanded metal foils, honeycombs, and so on embedded in some matrix material (prepreg resins and so on). In what follows, we will focus ourselves to periodic reciprocal media, as a model of woven carbon fiber composites and reinforcing wire meshes. For this media, we will adopt the more convenient notation shown in Fig. 1 to refer to the electric components of the TE and TM modes as $E_{\psi}$ and $E_{\zeta}$, respectively. We will next revisit two of the most well-known methods to analyze these media: the Holloway and the Wait models.

\section{A. Holloway's Model}

Holloway et al. [6] propose and analyze three different models for carbon-fiber composites. These are composed of 
layers characterized with a thickness $L$, with round fibers of diameter $D$ which repeat with a periodicity $P$ [6, Fig. 1]. The fibers have a permittivity $\varepsilon_{f}$ and permeability $\mu_{f}$ and are assumed to be surrounded by the matrix bulk material forming the remainder of each layer with $\varepsilon_{b}$ and $\mu_{b}$. The fiber composite layer is decomposed in several regions. In each of these regions, the effective permittivity tensor $\overline{\bar{\varepsilon}}$ is calculated as

$$
\overline{\bar{\varepsilon}}_{i j}=\varepsilon_{i j} \delta_{i j}
$$

with

$$
\begin{aligned}
\varepsilon_{z z}^{-1} & =(1-g) \varepsilon_{b}^{-1}+g \varepsilon_{f}^{-1} \\
\varepsilon_{y y} & =\varepsilon_{x x}=(1-g) \varepsilon_{b}+g \varepsilon_{f}
\end{aligned}
$$

and $g$ being a geometric parameter depending on the particular shape of the fibers. A similar formula could be obtained for the effective permeability tensor. Transmission matrix coefficients can be straightforwardly obtained from the stacking of layers with these anisotropic permittivity and permeability tensors rotated accordingly to the fiber orientation [16].

The model predictions are compared there with results obtained from finite element method (FEM) simulations. From them, the authors conclude that their so-called threelayer inhomogeneous model, referred hereafter as Holloway's model, provides the best results for frequencies in which $P / \lambda<1$. At frequencies below the skin depth of an equivalent conductive layer, the model matches the expected results. At higher frequencies, the model will still produce accurate results, when the conducting fibers are closely packed, and the proximity effects dominate over inductive and skin effects such as the ones discussed in Section III-B. This proximity effect is caused by conduction time-varying currents inside the wires which create nonnegligible eddy currents in their neighbors. Similar to the skin effect, this effectively increases their total impedance. This effect becomes more important when several layers of wires are stacked closely and has been studied in detail in the context of the transformer windings used in electric engineering [17], [18].

\section{B. Wait's Model}

Wait [5] obtains the following expression for the surface impedance presented by an array of wires. The assumptions made to obtain such formula are that there are no resonances within the wire or on its surface $(\lambda \gg a)$, and as long as the distance between wires compared with their radius is sufficiently high to avoid proximity effects, i.e., $P \gg a$

$$
Z_{s}=Z_{w} P+j \eta_{b} \frac{P}{\lambda_{b}} \log \frac{P}{\pi a} .
$$

The sheet impedance can be used with the transmission matrix formalism in the following way [4]:

$$
\left[\begin{array}{c}
E_{\psi}(a / 2) \\
H_{\zeta}(a / 2) \\
E_{\zeta}(a / 2) \\
H_{\psi}(a / 2)
\end{array}\right]=\left[\Phi_{i}\right]\left[\begin{array}{c}
E_{\psi}(-a / 2) \\
H_{\zeta}(-a / 2) \\
E_{\zeta}(-a / 2) \\
H_{\psi}(-a / 2)
\end{array}\right]
$$

with

$$
\left[\Phi_{i}\right]=\left[\begin{array}{cccc}
1 & 0 & 0 & 0 \\
0 & 1 & 0 & 0 \\
0 & 0 & 1 & 0 \\
0 & 0 & 1 / Z_{S} & 1
\end{array}\right]
$$

In [4], metallic meshes and expanded foils are modeled using (11). The stacking of several layers is a valid approach when then interactions between different wire grids can be neglected. This will be true if the wire grids are perpendicular; however, if several layers of aligned wire grids are stacked, the effective periodicity is reduced and this results in proximity effects not being negligible, invalidating the model.

\section{Validation With Fine-Structure Simulations}

In this section, we will discuss the validity of the presented models. To do so, the periodic microscopic pattern of several geometries is simulated with an FEM FD tool, Ansys HFSS, in the range between 0.1 and $100 \mathrm{GHz}$; and the results are compared with Holloway and Wait's predictions. Note that a TD simulator is not efficient performing these microscopic simulations as the space-step necessary to properly resolve these geometries would be approximately $1 \mu \mathrm{m}$, therefore imposing a time-step which does not allow us to obtain low-frequency results in an acceptable number of iterations. The structures have been chosen as representative of four common situations found in materials used in the industry: two wire meshes, one dense and one nondense, with no ohmic connection between different wires, an expanded metal foil with ohmic connections at the metal intersections, and a one-layer single-orientation CFC material. For all the cases considered, we will assume that the materials are embedded in free-space as the effect of resin materials (nonconductive and with a low permittivity) is negligible for fibers of the conductivity considered here.

\section{A. Loose Wire Mesh}

Let us start by modeling a loose wire mesh [Fig. 2(a)] consisting of two arrays of parallel wires and forming an angle of $89^{\circ}$. This panel was obtained from [4] (S1) and was modeled with two layers of wires having $a_{1}=a_{2}=50 \mu \mathrm{m}$, $\sigma=58 \mathrm{MS} / \mathrm{m}$, and $P_{1}=P_{2}=558 \mu \mathrm{m}$. In this case, wires are widely separated $(P / a \approx 11)$ and therefore we can expect the inductive effects to dominate. As expected, we obtain a good match between simulations and Wait's model (Fig. 3), with differences of less than $0.5 \mathrm{~dB}$. Holloway's model fails for this case as it does not take into account inductive effects, and proximity effects are negligible. Experimental data for the SE of this test case are also shown in Section VII compared with FDTD results found after Wait's prediction for the macroscopic S-parameter matrix.

\section{B. Expanded Foil}

The second test-case is an aluminum expanded foil [Fig. 2(b)], similar to the ones presented in [4]. In this case, 


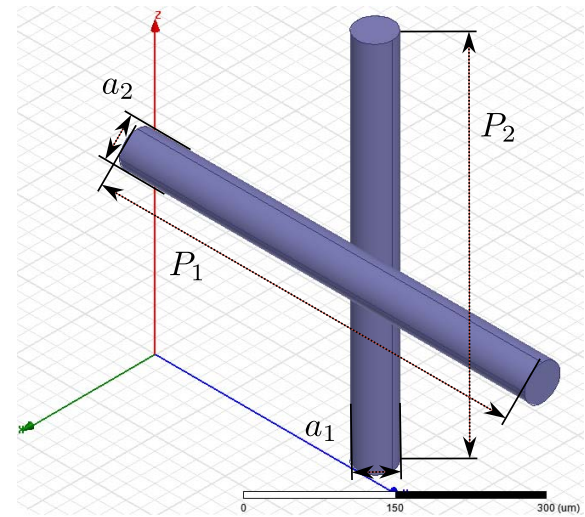

(a)

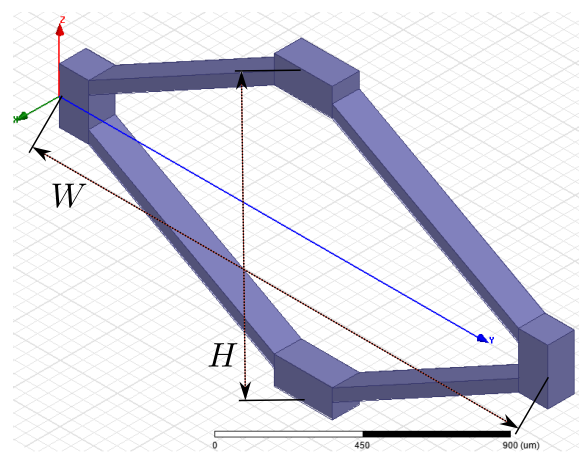

(b)

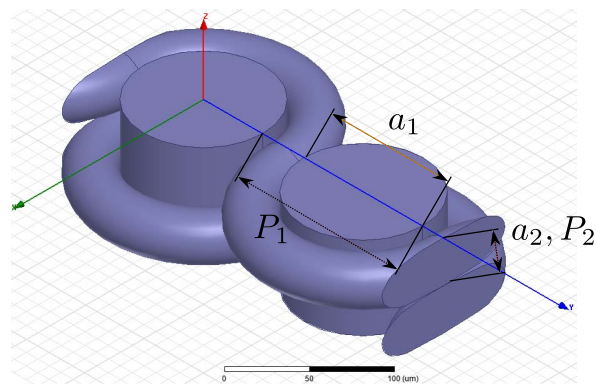

(c)

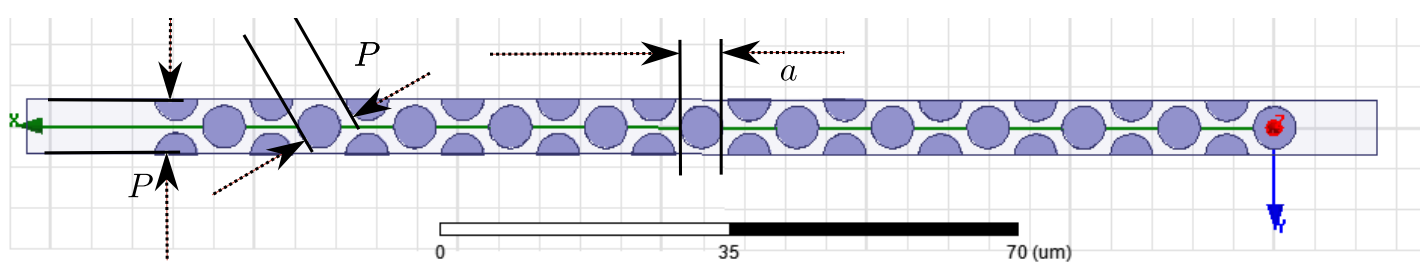

(d)

Fig. 2. Microscopic models employed for simulations. (a) Prepreg copper mesh of [4] (S1). (b) Aluminum expanded foil. (c) Stainless steel wire mesh. (d) CFC layer model composed of 24 sublayers.

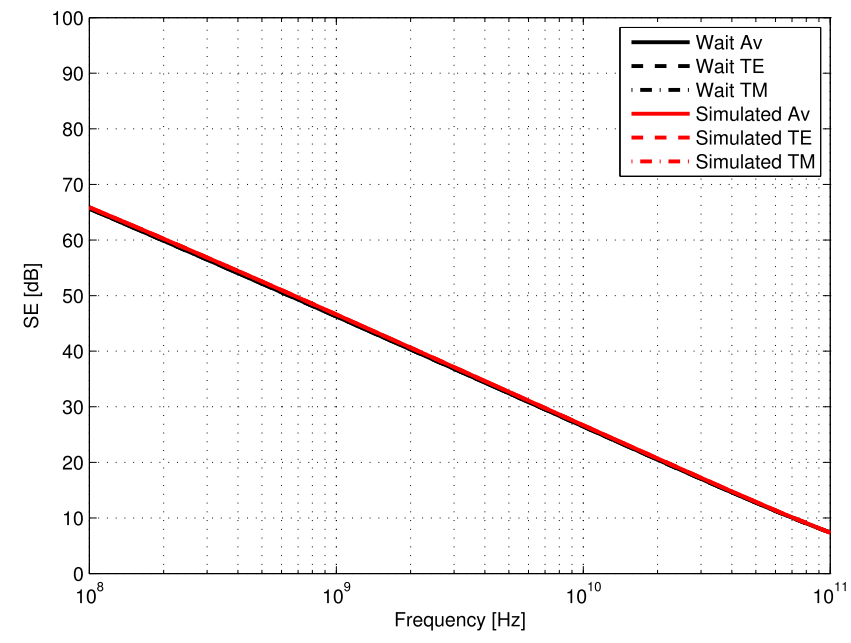

Fig. 3. SE results for the loose wire mesh configuration of Fig. 2(a). Holloway's model results are not shown for falling out of the scale.

we have three significant differences with respect to the previous case: intersection points now have ohmic connectivity, wire sections are not round, and the lines are forming an angle of $57.16^{\circ}$. For their incorporation into the analytical models, the rectangular cross sections of the expanded foil were substituted by area-equivalent round wires with $a=90 \mu \mathrm{m}$ and $\sigma=38 \mathrm{MS} / \mathrm{m}$. With this geometrical data, we can draw a unit cell with periodicities of $W=2129 \mu \mathrm{m}$ and $H=1259 \mu \mathrm{m}$. The periodicities of the two layers used in the models were $P=1089 \mu \mathrm{m}$. Similar to the case in Section IV-A, the Holloway's model does not provide good predictions, due to the lack of validity of the effective layer

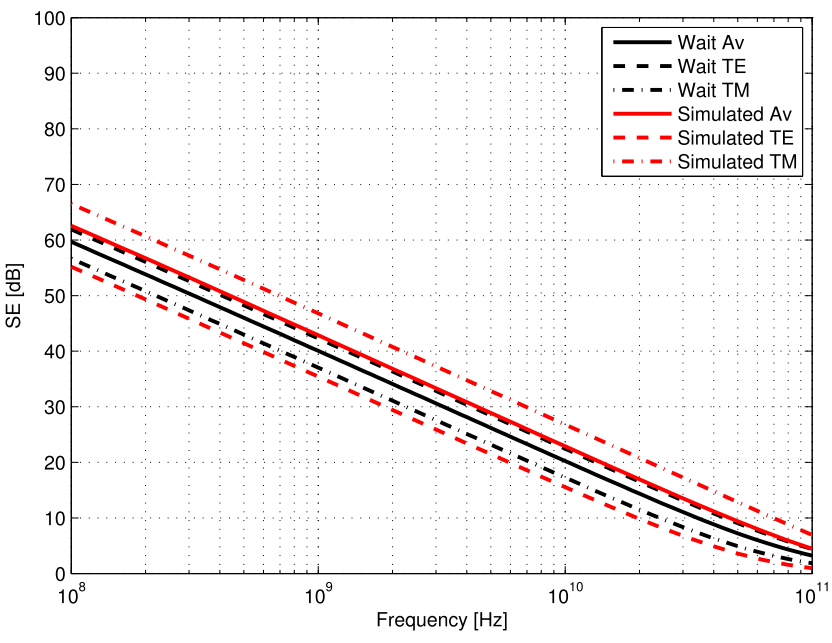

Fig. 4. SE results for the expanded foil of Fig. 2(b). Holloway's model results are not shown for falling out of the scale.

approximation (Fig. 4). Wait's model predicts an average SE about $3 \mathrm{~dB}$ lower, likely due to the overestimation of impedance in the wire intersections.

\section{Dense Wire Mesh}

We now aim to test the model with a dense stainless steel mesh [Fig. 2(c)] in which the $P / a \approx 1$. To model this setup, we use two perpendicular arrays of wires with $a_{1}=100 \mu \mathrm{m}, a_{2}=35 \mu \mathrm{m}, \sigma=37 \mathrm{kS} / \mathrm{m}, P_{1}=135 \mu \mathrm{m}$, and $P_{2}=35 \mu \mathrm{m}$. Results (Fig. 5) show some agreement between Wait and Holloway's models at low frequencies but fail at higher frequencies. A reason for this is that when the wires 


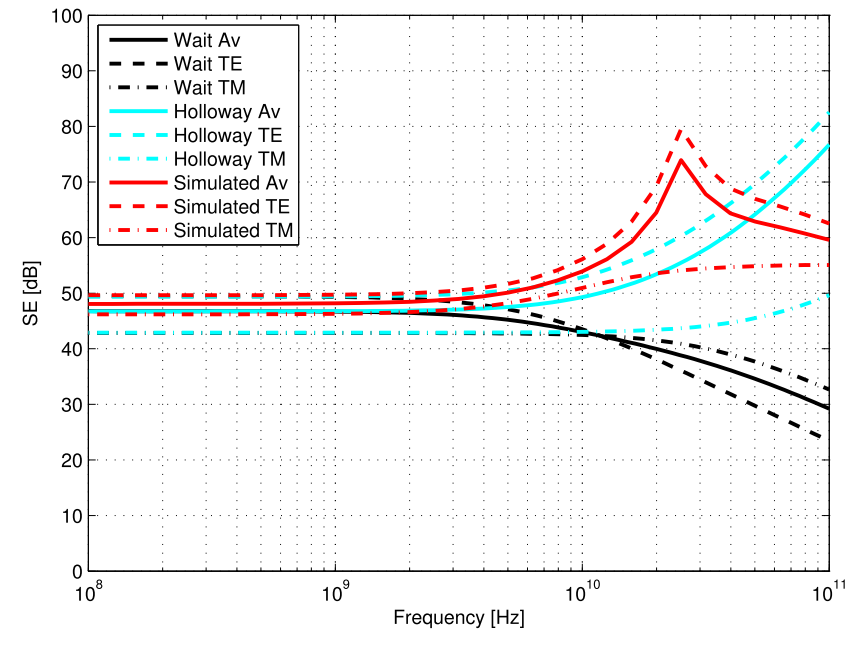

Fig. 5. SE results for the dense steel mesh configuration of Fig. 5.

are too close to each other, there is a dominance of the proximity effects, which are eventually overcome by the inductive behavior of the grid. This mixed behavior seems to indicate that possibly a hybrid model combining the Holloway's and Wait's approach can provide optimal results for this type of configurations.

\section{Carbon Fiber Composites}

Finally, woven CFC composite panels are among the most used materials in modern industry. These panels are created by the weaving of several sublayers of fibers with one or different orientations. Each of these sublayers is composed of tows, each containing thousands of fibers. The microscopic structure of a typical CFC layer [Fig. 2(d)] has been modeled for our simulation by stacking 24 sublayers of fibers with $a=5.2 \mu \mathrm{m}, \sigma=66.667 \mathrm{kS} / \mathrm{m}$, and $P=6.76 \mu \mathrm{m}$. Odd and even sublayers are displaced $P / 2$ in the $y$-axis, therefore leaving a distance of $P \sqrt{3} / 2$ between them. For this, we have just made simple assumptions based on the single fiber radius, and the volume density fiber provided by the manufacturer. Just a single-layer single-orientation model has been analyzed, though usual panels may have up to several tenths of layers with different orientations, making them difficult both to simulate and to measure. Simulation and model results (Fig. 6) show that, as we may expect, the proximity effects dominate not only because of the neighbor fibers in the same sublayer but due to the ones in the neighboring sublayers which have the same orientation. As a consequence, the field cannot penetrate more than a few microns into the $\mathrm{CFC}$ at high frequencies (Fig. 7). While all models agree at low frequencies, Holloway's matches simulated results with a deviation of approximately $2 \mathrm{~dB}$ in the range of frequencies studied. We can compare this result with the ones obtained for an equivalent $76-\mu \mathrm{m}$ bulk layer of material which presents a much larger deviation. We can also conclude that Wait's model is not effective to study this type of structures, due to the dominance of the proximity effects. It bears noticing that we have assumed the CFC to have a homogeneous stacked distribution of the carbon fibers, and we have neglected any

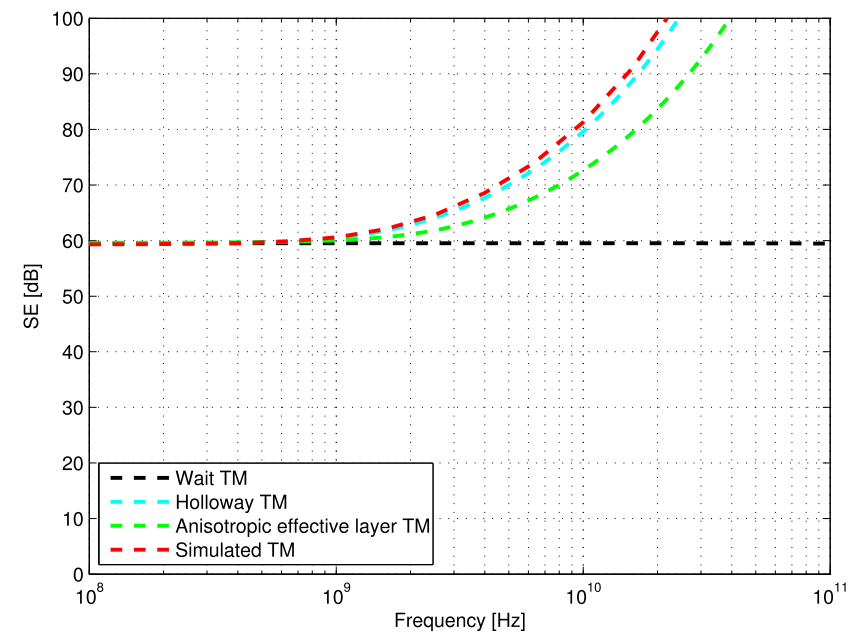

Fig. 6. SE results for the CFC [Fig. 2(d)]. TE mode is zero for all the cases.

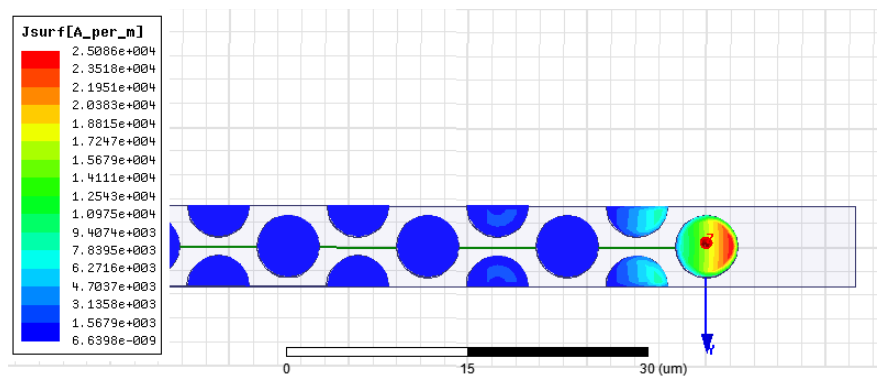

Fig. 7. Induced currents in the CFC [Fig. 2(d)] at $100 \mathrm{GHz}$.

weaving fabric geometrical features. In practice, the weaving will interrupt the spatial homogeneity of the fibers clustering them in warps with some separation among them, which will create an inductive point of entry of EM energy. This will compete with the shielding provided by each individual warp due to their internal proximity effect dominance.

\section{FINDING A TD MODEL}

Holloway and Wait's techniques described in the previous sections permit us to find the whole S-parameter matrices for different material arrangements starting from their microscopic structure. We can incorporate their behavior into numerical methods by means of NIBCs, as described in [10], or by the novel SGBCs introduced by Cabello et al. [14], [15]. The latter approach has proven to exhibit a superior late time stability compared with the NIBC method. However, the S-parameters must be first transformed into bulk constitutive complex permittivity and permeability effective parameters $\varepsilon_{\text {eff }}$ and $\mu_{\text {eff }}$, for some given thickness, to be used by SGBC.

Bulk parameter retrieval from S-parameters remains an active topic of research [19]. In this paper, we will just briefly summarize the procedure that we have adopted, based on the work of [20]. For this, let us assume for simplicity a nonmagnetic, isotropic, left-right symmetric $\left(S_{11}=S_{22}\right)$, and reciprocal material $\left(S_{12}=S_{21}\right)$. The relationship between the S-parameters under normal incidence and the effective 
permittivity can be expressed as

$$
\begin{aligned}
& S_{11}=R \frac{1-\psi^{2}}{1-R^{2} \psi^{2}} \\
& S_{21}=\frac{\left(1-R^{2}\right) \psi}{1-R^{2} \psi^{2}}
\end{aligned}
$$

with

$$
\begin{aligned}
& R=\frac{\eta_{\text {eff }}-\eta_{0}}{\eta_{\text {eff }}+\eta_{0}} \\
& \psi=e^{-j n_{\text {eff }} k_{0} t_{h}}
\end{aligned}
$$

where $k_{0}=\omega / c_{0}$ is the free-space wavenumber, $\eta_{0}=\left(\mu_{0} / \varepsilon_{0}\right)^{1 / 2}$ is the free-space impedance, and $\eta_{\text {eff }}=$ $\left(\mu_{\text {eff }} / \varepsilon_{\text {eff }}\right)^{1 / 2}$ that of the effective medium. $t_{h}$ stands for the material thickness. There is a degree of freedom to choose this parameter, which does not necessarily need to match the actual physical thickness. Different bulk constitutive parameters will be found for slabs with different $t_{h}$ values, all with the same S-parameters. From (12) and (13b), the procedure is as follows.

1) The effective impedance is found after some algebra by

$$
\eta_{\mathrm{eff}}= \pm \eta_{0} \sqrt{\frac{\left(1+S_{11}\right)^{2}-S_{21}^{2}}{\left(1-S_{11}\right)^{2}-S_{21}^{2}}}
$$

where according to [19], any choice of the sign is equally valid (some authors also use a passivity condition to keep just one of them).

2) The effective refractive index is next found by

$$
n_{\mathrm{eff}}=j \frac{\log \psi}{k_{0} t_{h}}=\left(j \frac{\log |\psi|}{k_{0}}-\frac{L \psi+2 \pi m}{k_{0}}\right) \frac{1}{t_{h}}
$$

with

$$
\psi=\frac{S_{21}}{1-S_{11} \Gamma} \text { and } \Gamma=\frac{\eta_{\mathrm{eff}}-\eta_{0}}{\eta_{\mathrm{eff}}+\eta_{0}} .
$$

Note that the refractive index admits infinite solutions for its real part depending on the integer number $m$, each one corresponding to a branch of the complex logarithm. This fact introduces an added difficulty that has attracted a lot of research efforts since the original works of [21] and [22].

3) The $\varepsilon_{\text {eff }}$ can now be found from (14) and (15); however, the assumption of a nonmagnetic material releases us from solving the branch problem, since $\varepsilon_{\text {eff }}$ can be directly found by

$$
\begin{aligned}
& \varepsilon_{\text {eff }}(\omega)=\eta_{\text {eff }}^{2} / \mu_{0} \\
& \mu_{\text {eff }}(\omega)=\mu_{0} .
\end{aligned}
$$

The time-domain version of the frequency-dependent $\varepsilon_{\text {eff }}$ can now be easily found by employing a VF procedure [23] to obtain an $N$ th-order pole-residue partial fraction expansion

$$
\varepsilon_{\text {eff }}(\omega)=\varepsilon_{\infty}+\sum_{k=1}^{N} \frac{R_{k}}{j \omega-p_{k}}
$$

which can include complex or purely real pole-residues. In case that these are complex, the complex conjugat of the pair must also appear in the expansion. Now, following the methodology of [24], we introduce a set of polarization currents $\vec{J}_{k}(\omega)$ each one related to the E-field in FD and in $\mathrm{TD}$, respectively, by

$$
\begin{aligned}
\vec{J}_{k}(\omega) & =\frac{j \omega R_{k}}{j \omega-p_{k}} \vec{E}(\omega) \\
\frac{d \vec{J}_{k}(t)}{d t}-p_{k} \vec{J}_{k}(t) & =R_{k} \frac{d \vec{E}(t)}{d t}
\end{aligned}
$$

which are plugged into Maxwell equations in TD to end up with

$$
\begin{aligned}
-\nabla \times \vec{E} & =\mu_{0} \frac{\partial \vec{H}}{\partial t} \\
\nabla \times \vec{H} & =\sum_{k=1}^{N} \vec{J}_{k}+\varepsilon_{\infty} \frac{\partial \vec{E}}{\partial t} .
\end{aligned}
$$

\section{IMPLEMENTATION INTO SGBC}

The SGBC method introduced in [14] was born as a robustly stable subcell alternative of NIBC to find the tangential electric fields at each side of the thin slab, as a function of the magnetic fields in the adjacent host medium. It combines two methods: 1) a CNTD method in 1-D, to find the transversal EM solution within the slab, which is finely meshed to account for the fields variation inside and 2) the usual 3D Yee-FDTD method to solve the exterior problem coarsely discretized according to the desired resolution. A proper HIE algorithm is used to connect both methods at the slab boundaries. The unconditionally stable nature of CNTD and the natural HIE scheme we employ allows us to choose the time-step to meet the stability criterion just depending on the outer coarser mesh size, without compromising the computational affordability of the method.

The method described in [14] for nondispersive media can be extended to dispersive ones [15], as described next in a simplified manner. For this, let us assume a nonmagnetic pure homogeneous SGBC medium, meshed with a uniform spacestep $\Delta_{\text {fine. }}$ To find the CNTD scheme used inside, we start from the usual FDTD scheme for (20) and (21) to find

$$
\begin{aligned}
J_{i, k}^{n}= & \kappa_{k} J_{i, k}^{n-1}+\beta_{k}\left(E_{i}^{n}-E_{i}^{n-1}\right) \quad \forall k=1, \ldots, N \\
E_{i}^{n+1}= & C_{a} E_{i}^{n}+C_{b}\left(H_{i-\frac{1}{2}}^{n+\frac{1}{2}}-H_{i+\frac{1}{2}}^{n+\frac{1}{2}}\right) \\
& -C_{J} \operatorname{Re} \sum_{k=1}^{N}\left(1+\kappa_{k}\right) J_{i, k}^{n} \\
H_{i+\frac{1}{2}}^{n+\frac{1}{2}}= & D_{a} H_{i+\frac{1}{2}}^{n-\frac{1}{2}}+D_{b}\left(E_{i}^{n}-E_{i+1}^{n}\right)
\end{aligned}
$$

where the coefficients $C_{a}, C_{b}, D_{a}, D_{b}, C_{J}, \beta_{k}$, and $\kappa_{k}$ are

$$
\begin{aligned}
C_{a} & =\frac{2 \varepsilon_{\infty}+\Lambda-\sigma \Delta t}{2 \varepsilon_{\infty}+\Lambda+\sigma \Delta t} \quad C_{b}=\frac{2 \Delta t / \Delta_{\text {fine }}}{2 \varepsilon_{\infty}+\Lambda+\sigma \Delta t} \\
D_{a} & =1 \\
D_{b} & =\frac{2 \Delta t / \Delta_{\text {fine }}}{2 \mu_{0}} \quad C_{J}=\frac{C_{b}}{2} \Delta_{\text {fine }}
\end{aligned}
$$




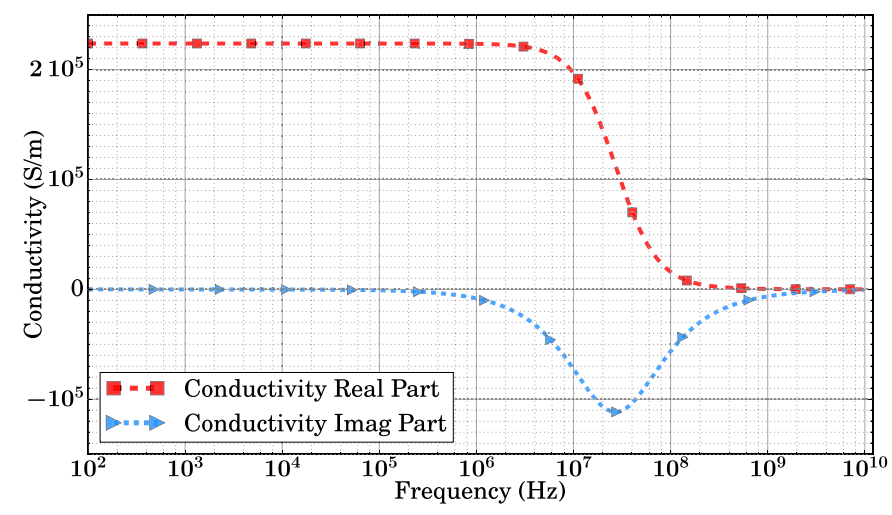

Fig. 8. Equivalent dielectric bulk parameters for the material of Fig. 2(a) with a thickness of $100 \mu \mathrm{m}$.

with

$$
\begin{aligned}
\Lambda & =\operatorname{Re} \sum_{k=1}^{N} \beta_{k} \Delta t \\
\beta_{k} & =\frac{R_{k}}{1-p_{k} \Delta t / 2} \quad \kappa_{k}=\frac{1+p_{k} \Delta t / 2}{1-p_{k} \Delta t / 2} .
\end{aligned}
$$

Now, the CNTD algorithm [15] is built from (23), by colocating the $\mathrm{H}$ - and E-fields at integer time-steps, with the help of a time average for the right-hand side to yield

$$
\begin{aligned}
E_{i}^{n+1}= & C_{a} E_{i}^{n}+\frac{C_{b}}{2}\left(H_{i-\frac{1}{2}}^{n+1}-H_{i+\frac{1}{2}}^{n+1}+H_{i-\frac{1}{2}}^{n}-H_{i+\frac{1}{2}}^{n}\right) \\
& -C_{J} \operatorname{Re} \sum_{k=1}^{N}\left(1+\kappa_{k}\right) J_{k}^{n} \\
H_{i+\frac{1}{2}}^{n+1}= & D_{a} H_{i+\frac{1}{2}}^{n}+\frac{D_{b}}{2}\left(E_{i}^{n}-E_{i+1}^{n}+E_{i}^{n+1}-E_{i+1}^{n+1}\right)
\end{aligned}
$$

and leaving (23a) untouched.

Now extracting $H^{n+1}$ from (27) and inserting it into (26), a fully consistent algorithm with the space positions of $E$ and $H$ staggered in space, as in the usual Yee-Scheme, is yielded, but evaluated at colocated integer time-steps. After some algebra, a tridiagonal system of equations is found for the $E$-fields

$$
a E_{i-1}^{n+1}+b E_{i}^{n+1}+c E_{i+1}^{n+1}=d_{i}^{n}
$$

with

$$
\begin{aligned}
a= & -\frac{C_{b} D_{b}}{4}=c \quad b=1-a-c \\
d_{i}^{n}= & \frac{C_{b} D_{b}}{4}\left(E_{i-1}^{n}+E_{i+1}^{n}\right)+\left(C_{a}-\frac{C_{b} D_{b}}{2}\right) E_{i}^{n} \\
& +\frac{C_{b}}{2}\left(1+D_{a}\right)\left(H_{i-\frac{1}{2}}^{n}-H_{i+\frac{1}{2}}^{n}\right)-C_{J} J_{i}^{n} .
\end{aligned}
$$

Note that the $H$-fields are advanced explicitly by (27) from the $E$-fields. The HIE conditions to connect the CNTD equations, used inside the slab, to the usual FDTD equations, used in the surrounding media, are built in the same manner given in [14] (not given here for simplicity).
TABLE I

TEnth-ORder Vector-Fitted Poles And Residues FOR THE MATERIAL OF FIG. 2(a)

\begin{tabular}{|l|l|}
\hline Residue & Pole \\
\hline \hline$+2.23 e+05$ & $4.99 e-02$ \\
\hline$-1.32 e+00$ & $8.56 e+03$ \\
\hline$-2.22 e+05$ & $1.70 e+08$ \\
\hline$-7.00 e+02$ & $3.37 e+09$ \\
\hline$+1.13 e-03-2.42 e-04 j$ & $6.46 e+02-7.74 e+04 j$ \\
\hline$+1.13 e-03+2.42 e-04 j$ & $6.46 e+02+7.74 e+04 j$ \\
\hline$+1.10 e-03+7.65 e-05 j$ & $2.94 e+02-1.29 e+05 j$ \\
\hline$+1.10 e-03-7.65 e-05 j$ & $2.94 e+02+1.29 e+05 j$ \\
\hline$-2.32 e-03-4.85 e-04 j$ & $1.75 e+03-2.09 e+05 j$ \\
\hline$-2.32 e-03+4.85 e-04 j$ & $1.75 e+03+2.09 e+05 j$ \\
\hline$-6.25 e-04-9.00 e-04 j$ & $2.76 e+04-3.01 e+05 j$ \\
\hline$-6.25 e-04+9.00 e-04 j$ & $2.76 e+04+3.01 e+05 j$ \\
\hline
\end{tabular}

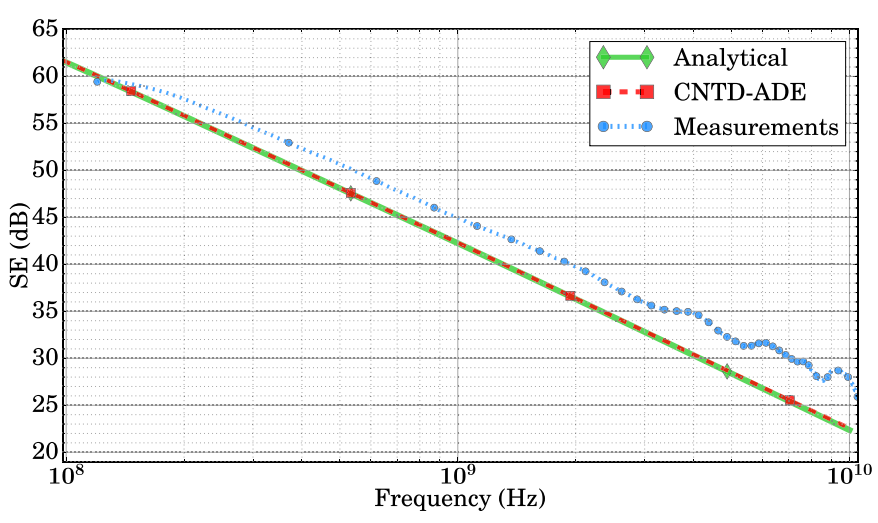

Fig. 9. Comparison of the TE SE for the material of Fig. 2(a) and experimental results from [4] (S1).

\section{VALIDATION}

A canonical validation of this method is presented here. It computes the $\mathrm{SE}$ of the indefinite thin panel of the prepeg copper mesh [Fig. 2(a)] previously analyzed in Section IV-A. The constitutive parameters of the equivalent negative-permittivity material have been extracted from the S-parameters found with Wait's model (Fig. 8), assuming a thickness $t_{h}=100 \mu \mathrm{m}$, and vector-fit with a tenth-order poleresidue expansion (see Table I). We have simulated a thin-slab of this material under plane-wave TE incidence, with the HIE CNTD method described previously, taking ten cells along its thickness. Results for its SE are shown in Fig. 9. They perfectly match with the analytical ones and reasonably agree with experimental measurements from [4] (S1).

\section{CONCLUSION}

In this paper, we have presented a full road map to start from the microscopic nature of a composite multilayered thin panel and end into a macroscopic TD model to be simulated by FDTD.

In the first part, we have revisited Holloway's and Wait's model and discussed some of their limitations, taking profit of low-level simulations of their fine structure. While both methods reasonably agree in the asymptotic low-frequency regime, differences appear at high frequency. We have shown that for nondense structures, Holloway's model does not 
replicate the simulated behavior at high frequencies, whereas Wait's assumptions provide a more realistic model. On the other hand, for structures densely stacked along the panel thickness, like the CFC test-case (with the homogeneous assumption), the material has a classical conductorlike skineffect behavior, properly predicted by Holloway's model and falling out of Wait's hypotheses. Finally, for intermediate structures, the behavior is a combination of both models and still requires a deeper study.

In the second part of this paper, we have extended the SGBC procedure to deal with the dispersive nature of thin panels, assuming that their scattering parameters are found with the techniques given in the first part. A parameter extraction procedure has been presented, and a time-domain implementation of the dispersive constitutive parameters has been described. For this, the CNTD method used by SGBC has been extended. Finally, the whole road map has been applied to one of the test cases used in the first part to prove the feasibility of this approach.

\section{ACKNOWLEDGMENT}

The authors would like to thank A. R. Lora for helping with the drawing of the thin-panel models used in Section IV.

\section{REFERENCES}

[1] A. Tamburrano, D. Desideri, A. Maschio, and M. S. Sarto, "Coaxial waveguide methods for shielding effectiveness measurement of planar materials up to $18 \mathrm{GHz}$," IEEE Trans. Electromagn. Compat., vol. 56 , no. 6, pp. 1386-1395, Dec. 2014.

[2] H. Vasquez, L. Espinoza, K. Lozano, H. Foltz, and S. Yang, "Simple device for electromagnetic interference shielding effectiveness measurement," IEEE EMC Soc. Newslett., no. 220, pp. 62-68, Winter 2009. [Online]. Available: http://www.emcs.org/acstrial/newsletters/winter09/

[3] M. S. Sarto and A. Tamburrano, "Innovative test method for the shielding effectiveness measurement of conductive thin films in a wide frequency range," IEEE Trans. Electromagn. Compat., vol. 48, no. 2, pp. 331-341, May 2006

[4] M. S. Sarto, S. Greco, and A. Tamburrano, "Shielding effectiveness of protective metallic wire meshes: EM modeling and validation," IEEE Trans. Electromagn. Compat., vol. 56, no. 3, pp. 615-621, Jun. 2014.

[5] J. R. Wait, "Reflection at arbitrary incidence from a parallel wire grid," Appl. Sci. Res., Sec. A, vol. 4, no. 1, pp. 393-400, 1955. [Online] Available: http://dx.doi.org/10.1007/BF02316501

[6] C. L. Holloway, M. S. Sarto, and M. Johansson, "Analyzing carbonfiber composite materials with equivalent-layer models," IEEE Trans. Electromagn. Compat., vol. 47, no. 4, pp. 833-844, Nov. 2005.

[7] V. Volski and G. A. E. Vandenbosch, "Full-wave electromagnetic modelling of fabrics and composites," Compos. Sci. Technol., vol. 69, no. 2, pp. 161-168, 2009. [Online]. Available: http://www.sciencedirect.com/science/article/pii/S0266353808003825

[8] V. Préault, R. Corcolle, L. Daniel, and L. Pichon, "Effective permittivity of shielding composite materials for microwave frequencies," IEEE Trans. Electromagn. Compat., vol. 55, no. 6, pp. 1178-1186, Dec. 2013.

[9] D. A. Frickey, "Conversions between S, Z, Y, H, ABCD, and T parameters which are valid for complex source and load impedances," IEEE Trans. Microw. Theory Techn., vol. 42, no. 2, pp. 205-211, Feb. 1994.

[10] M. S. Sarto, "A new model for the FDTD analysis of the shielding performances of thin composite structures," IEEE Trans. Electromagn. Compat., vol. 41, no. 4, pp. 298-306, Nov. 1999.

[11] B. Gustavsen, "Improving the pole relocating properties of vector fitting," IEEE Trans. Power Del., vol. 21, no. 3, pp. 1587-1592, Jul. 2006.

[12] G. Kobidze, "Implementation of collocated surface impedance boundary conditions in FDTD," IEEE Trans. Antennas Propag., vol. 58, no. 7, pp. 2394-2403, Jul. 2010.

[13] V. Nayyeri, M. Soleimani, and O. M. Ramahi, "Modeling graphene in the finite-difference time-domain method using a surface boundary condition," IEEE Trans. Antennas Propag., vol. 61, no. 8, pp. 4176-4182, Aug. 2013.
[14] M. R. Cabello, "A hybrid Crank-Nicolson FDTD subgridding boundary condition for lossy thin-layer modeling," IEEE Trans. Microw. Theory Techn., vol. 65, no. 5, pp. 1397-1406, May 2017

[15] M. R. Cabello, L. D. Angulo, A. R. Bretones, R. G. Martin, S. G. Garcia, and J. Alvarez, "A novel subgriding scheme for arbitrarily dispersive thin-layer modeling," in IEEE MTT-S Int. Microw. Symp. Dig., May 2017, pp. 266-268.

[16] S. J. Orfanidis. (2002). Electromagnetic Waves and Antennas. Accessed: Dec. 18, 2017. [Online]. Available: http://www.ece.rutgers. edu/orfanidi/ewa/

[17] P. L. Dowell, "Effects of eddy currents in transformer windings," Proc. Inst. Electr. Eng., vol. 113, no. 8, pp. 1387-1394, Aug. 1966.

[18] D. C. Meeker, "An improved continuum skin and proximity effect model for hexagonally packed wires," J. Comput. Appl. Math., vol. 236, no. 18, pp. 4635-4644, 2012.

[19] S. Arslanagić, "A review of the scattering-parameter extraction method with clarification of ambiguity issues in relation to metamaterial homogenization," IEEE Antennas Propag. Mag., vol. 55, no. 2, pp. 91-106, Apr. 2013.

[20] X. Chen, T. M. Grzegorczyk, B.-I. Wu, J. Pacheco, Jr., and J. A. Kong, "Robust method to retrieve the constitutive effective parameters of metamaterials," Phys. Rev. E, Stat. Phys. Plasmas Fluids Relat. Interdiscip. Top., vol. 70, p. 016608, 2004. [Online]. Available: http://link.aps.org/doi/10.1103/PhysRevE.70.016608

[21] A. M. Nicolson and G. F. Ross, "Measurement of the intrinsic properties of materials by time-domain techniques," IEEE Trans. Instrum. Meas. vol. IM-19, no. 4, pp. 377-382, Nov. 1970.

[22] W. B. Weir, "Automatic measurement of complex dielectric constant and permeability at microwave frequencies," Proc. IEEE, vol. 62, no. 1, pp. 33-36, Jan. 1974.

[23] B. Gustavsen and A. Semlyen, "Rational approximation of frequency domain responses by vector fitting," IEEE Trans. Power Del., vol. 14 no. 3, pp. 1052-1061, Jul. 1999.

[24] M. Han, R. W. Dutton, and S. Fan, "Model dispersive media in finite-difference time-domain method with complex-conjugate poleresidue pairs," IEEE Microw. Wireless Compon. Lett., vol. 16, no. 3, pp. 119-121, Mar. 2006.

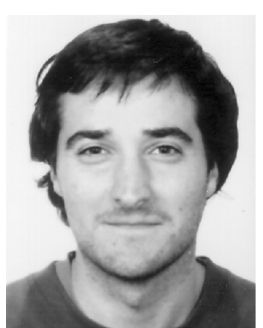

Luis Diaz Angulo received the M.Sc. degree in physics, Ph.D. degree, and M.Sc. degree in electronics engineering from the University of Granada, Granada, Spain, in 2008, 2014, and 2015, respectively.

$\mathrm{He}$ is currently an Interim Teacher with the Department of Electromagnetism, University of Granada. His current research interests include the time-domain numerical methods applied to electromagnetism, especially discontinuous Galerkin and finite-difference methods, and the application of numerical methods in EMC.

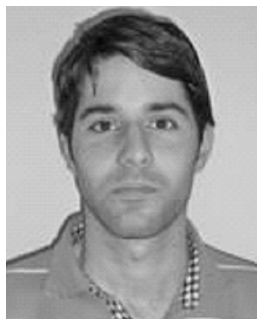

Miguel Ruiz Cabello was born in Granada, Spain. He received the B.Sc. and M.Sc. degrees in physics from the University of Granada, Granada, in 2008 and 2010, respectively, where he is currently pursuing the Ph.D. degree in physics at the Department of Electromagnetism and Matter Physics with a focus on HPC time-domain solvers for electromagnetic analysis and design.

$\mathrm{He}$ has participated in the 7PM EU project HIRF-SE as a developer of the SEMBA-UGRFDTD solver.

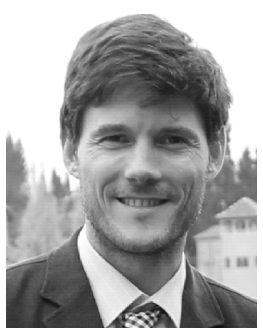

Jesus Alvarez (M'00) was born in Leon, Spain. He received the M.Sc. degree from the University Carlos III of Madrid, Getafe, Spain, in 2008, and the Ph.D. degree from the University of Granada, Granada, Spain, in 2013

Since 2006, he has been with Airbus Defence and Space, Toulouse, France, as an RCS, Antenna, and EMC Engineer. His current research interests include computational electrodynamics in the time domain, method of moments applications on electromagnetic compatibility, antennas, and RADAR cross sections. 


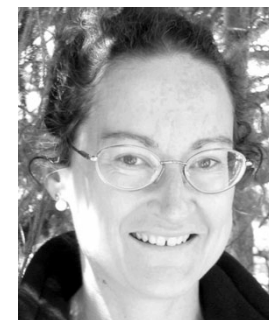

Amelia Rubio Bretones (SM'08) was born in Granada, Spain. She received the Ph.D. degree in physics from the University of Granada, Granada, in 1988.

Since 1985, she has been with the Department of Electromagnetism, University of Granada, where she has been a Full Professor since 2000. Her current research interests include numerical techniques for applied electromagnetics with an emphasis on timedomain techniques such as finite-difference timedomain and hybrid techniques.

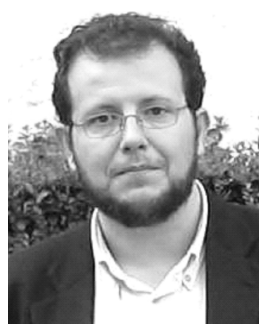

Salvador G. García (M'93-SM'14) received the M.S. and Ph.D. degrees (Hons.) in physics from the University of Granada, Granada, Spain, in 1989 and 1994, respectively.

In 1999, he joined the Department of Electromagnetism and Matter Physics, University of Granada, as an Assistant Professor, where he became a Ful Professor in 2016. He has authored or co-authored over 80 refereed journal papers and book chapters and led several national and international projects. His current research interests include computational electromagnetics, electromagnetic compatibility, RCS, and antenna design. 